\title{
ASSESSMENT OF HAZARD PERCEPTION LATENCIES USING REAL LIFE AND ANIMATED TRAFFIC HAZARDS: COMPARISON OF NOVICE AND EXPERIENCED DRIVERS
}

\author{
Nebi Sümer, ${ }^{1}$ Ayça Berfu Ünal, ${ }^{2}$ \& Ahmet Birdal ${ }^{2}$ \\ ${ }^{1}$ Department of Psychology \\ Middle East Technical University \\ Ankara, Turkey \\ E-mail: nsumer@metu.edu.tr \\ ${ }^{2}$ Psychotechnical Systems Group \\ Meteksan Sistem A.Ş. \\ Ankara, Turkey
}

\begin{abstract}
Summary: High accident risk of novice and inexperienced drivers is associated with their poor hazard perception ability. In past studies, hazard perception latencies (reaction time) were measured using hazards in simulated traffic environments, scenario-based video clips, or photographs, but rarely with real-life traffic situations. We developed two different measures to assess hazard perception ability (1) video clips of hazards recorded in real-life traffic settings and (2) the video clips of animated hazards. We compared these two measures in terms of their power in discriminating between novice and experienced drivers. Novice $(\mathrm{N}=43)$ and experienced drivers $(\mathrm{N}=65)$ were admisistered computerbased Turkish Hazard Perception Tests consisting of 40 real traffic and animated video clips of hazards. Results revealed that although experienced drivers detected the hazards relatively earlier than the novices on average, the difference between the groups was not statistically significant for both real-traffic and video clips. Examination of the group differences on each item suggested video clips reflecting actual traffic situations discriminate novice and experienced drivers better than animated clips. Content analyses of the clips that significantly discriminated groups revealed that novice drivers have difficulty in detecting hazards resulting from an unexpected or suddent violation of road users. It seemed that lack of experience in anticipating the other road users' violations creates a critical vulnerability for the safety of novice drivers.
\end{abstract}

\section{INTRODUCTION}

Young and novice drivers are more likely to be involved in road traffic accidents in the period immediately after they have been licenced (Mayhew, Simpson, \& Pak, 2003). The high accident risk of novice and inexperienced drivers is associated mainly with their poor hazard perception (HP) ability (see Deery, 1999), which taps proccesses regarding road-scanning strategies and anticipatory thinking about the risk, hazards, and oncoming events on the road. Past research indicated that, compared to experienced drivers, novice drivers fail in each stage of the hazard perception process, including effective scanning of the road, detecting potential hazards, and taking the most effective action to prevent a crash (Deery, 1999; Grayson \& Groeger, 2000). Recently, Wallis and Horswill (in press) showed that for novice drivers, higher thresholds of 
danger must be present before they identify a situation as hazardous, rather than failing to discriminate hazardous situations from non-hazardous ones.

Past studies on the attentional processes in driving demonstrated that, compared to experienced drivers, novices tend to attend more closely to the front of the vehicle when there is more traffic stimuli on the road (Falkmer \& Gregersen, 2001), they are ineffective at spotting hazards occurring in the distance (Brown, 1982), they fail to control and search the sides of the road (McKnight \& McKnight, 2003), and they fixate on irrelevant objects in traffic, using an inefficient visual search. Furthermore, novices were found to be unsuccessful in predicting the potential outcomes of other drivers' actions, which may prevent them from taking necessary hazard avoidance alternatives as quickly as possible (Bjornskau \& Sagberg, 2005).

Since hazard avoidance is central to decreasing crash risk, past studies mainly focused on HP latency (reaction time) measures. Past studies were not fully consistent regarding the differences between experienced and novice drivers on HP latency measures. While some found that young or novice drivers were significantly late in their responses in spotting hazardous situations (e.g., McKenna and Crick, 1997), other studies could not find significant differences (e.g., Sagberg \& Bjornskau, 2006). These inconclusive results may stem from the differences in the measures utilized in these studies. HP ability has been commonly measured by presenting drivers with footage of hazardous traffic situations recorded from the driver's point of view and asking them to respond as soon as they spot a hazard. However, the materials used in these measures vary from simulated traffic environments, to scenario-based video clips, to still photographs.

Researchers rarely used genuine traffic scenes reflecting the real traffic environment, with a few exceptions (Sagberg \& Bjornskau, 2006; Wallis \& Horswill, in press). Furthermore, different reaction time measures and their content, including various types of road hazards, were not compared in terms of their predictive or discriminating power in past studies. In this study, we first developed two different measures to assess HP ability: (1) video clips of hazards recorded in real-life traffic settings and (2) video clips of animated hazards. Second, we compared these two measures in terms of their power in discriminating between novice and experienced drivers. Finally, we aimed to identify the specific types of hazards and dangers in the Turkish driving environment that novice drivers have higher detection failure for than experienced drivers.

\section{METHOD}

\section{Participants}

Participants were novice $(\mathrm{N}=43)$ and experienced $(\mathrm{N}=65)$ drivers who were selected depending on their actual years of driving experience and annual $\mathrm{km}$ driven. Drivers who had been actively driving less than a year or who were recently licensed were categorized under the novice driver group, and those who had been actively driving more than 3 years with a weekly km driven over $200 \mathrm{~km}$ were categorized as experienced drivers. Mean years of active driving was $11.28(\mathrm{SD}=$ 5.26) for experienced drivers, and $.32(\mathrm{SD}=.47)$ for novice drivers. The sample consisted of male drivers only and the mean age was $27.85(\mathrm{SD}=7.27)$.

\section{Materials and Procedure}

Turkish Hazard Perception Test (T-HPT). The T-HPT is a computer-based test of HP ability composed of two sections, one including actual traffic video clips and the other including 
animated traffic scenes. The first section was constructed for the purpose of generating a reaction time latency measurement consisting of the common road hazards and traffic conflicts in Turkey. Considering that the scenario-based traffic scenes could not completely mirror the complexity of the actual traffic environment, actual traffic flows were recorded and used in the T-HPT. Similar to previous HP tests, recording was done from the driver's point of view using a handy camera stabilized on the windshield of a car. All recordings were taken from real traffic flows in different times within a day in major cities of Turkey.

A total of 60 hours of video footage was recorded on different roads in Turkey, and 266 scenes were selected. Short video clips that varied between 20 seconds to 40 seconds in length were prepared by screening this video-footage. The onset time and place of the hazards vary in each clip. Initially, the constructed clips were classified under broad categories using the Annual Traffic Statistics about the causes of accidents in Turkey, such as hazards occurring in junctions, overtaking hazards, close following hazards, etc. These items were further examined and rated by a panel consisting of 8 raters trained by researchers using a 10-point Likert-type scale $(10=$ very hazardous, $1=$ not hazardous). Of the 266 scene, 27 items with the highest ratings were selected as the test items (see Figure 1 for a sample screenshot). The panel used criteria similar to those suggested by Sexton (2001) in rating the hazard clips.

In addition to real-life traffic scenes, 10 animated scenes of video clips were created by simulating the actual traffic flow and complexity of road stimuli (see Figure 2). Scenarios that included at least one clear, developing hazard were produced considering the common types of hazards seen in Turkish traffic for animated videos. Each animated video lasted 30 seconds.

Drivers were administered a total of 40 real-life and animated video clips; three of them had no hazards for control purposes. Participants were clearly informed about what hazards meant and how they could response using a button connected to a computer with the help of three trial clips. If they failed to understand how to respond to a hazard, trials were repeated until they fully mastered the instructions. Drivers were allowed to give five responses at most. Hazard response windows were created tapping the onset and offset of each hazard. Each response (reaction time) was scored with reference to where it was given in the hazard response areas.

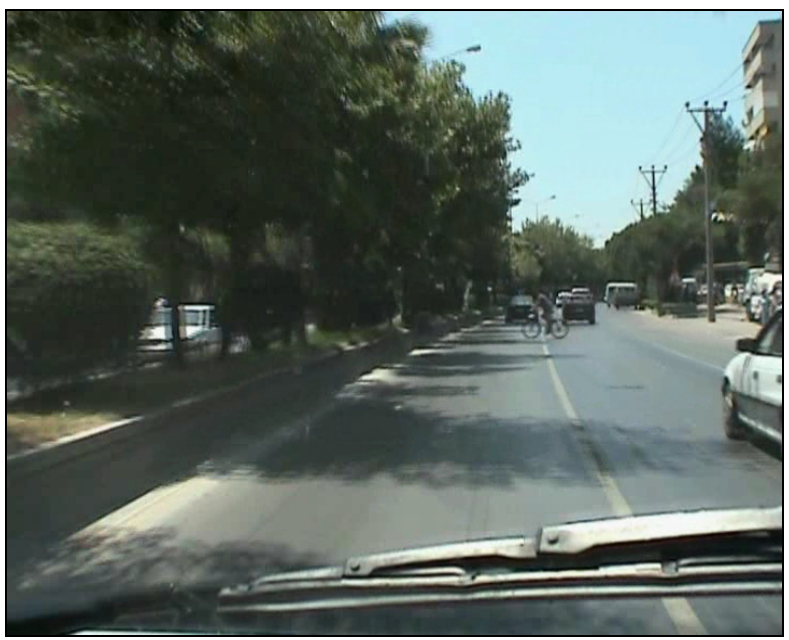

Figure 1. Sample screenshot from a real-life clip 


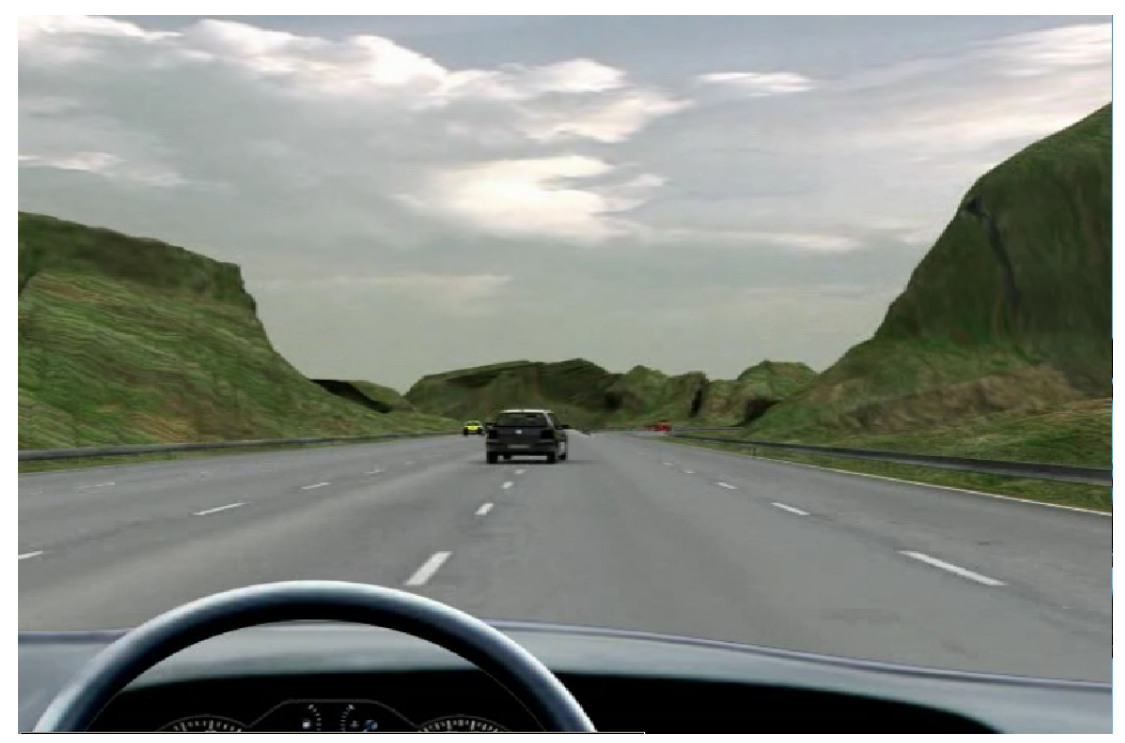

Figure 2. Sample screenshot from an animated clip

\section{RESULTS}

In order to examine the discriminating power of the real-traffic and animated video clips between experienced and novice drivers, separate analysis of covariance (ANCOVA) were run controlling for age on the mean scores of the measures. Results revealed that although experienced drivers detected the hazards relatively earlier than the novices on average, the difference between the groups was not statistically significant for both real-traffic video clips $(F(1,95)=2.45$, ns $)$ and animated clips $(F(1,95)=2.25, \mathrm{~ns})$. We also compared the two groups on the scores of each video clip using a series of ANCOVA controlling for age. As seen in Table 1, the results indicated that the scores of novice and experienced drivers were significantly or marginally significantly different $(<.10)$ from each other in nine of the real-life clips and in four of the animated clips. In these clips, mean response frames of experienced drivers were lower than novices, indicating that they responded earlier and closer to the onset of the hazardous situations.

Explained variances were higher among real-life clips, with more items reaching toward significance as compared to animated clips. In addition, the frame difference between the groups was over 100 frames in four of the real traffic clips, indicating that the responses of novice drivers were about four seconds late compared to experienced drivers. These findings suggest that video clips reflecting actual traffic situations discriminate novice and experienced drivers better than animated clips.

As seen in Table 2, examination of the content analyses of the clips that significantly discriminated groups revealed that the majority of these clips included a hazard or conflict resulting from an unexpected or suddent violation of other road users. It seemed that lack of experience in anticipating the other road users' violations created a critical vulnerability for the safety of novice drivers. 
Table 1. ANCOVA Results on Hazard Clips

\begin{tabular}{|c|c|c|c|c|c|}
\hline \multirow[t]{2}{*}{ Hazard Clips } & \multirow[t]{2}{*}{$\mathbf{F}$} & \multirow[t]{2}{*}{$\mathbf{P}$} & \multicolumn{2}{|c|}{ Mean Response Frame } & \multirow{2}{*}{$\begin{array}{c}\text { Frame } \\
\text { Difference }\end{array}$} \\
\hline & & & Novice Drivers & Experienced Drivers & \\
\hline Real-life video & & & & & \\
\hline 1 & 2.99 & .10 & 349 & 311 & 38 \\
\hline 2 & 8.62 & .01 & 553 & 501 & 52 \\
\hline 3 & 4.23 & .05 & 322 & 309 & 13 \\
\hline 4 & 2.69 & .10 & 230 & 208 & 22 \\
\hline 5 & 4.75 & .05 & 483 & 374 & 109 \\
\hline 6 & 2.05 & .10 & 591 & 489 & 102 \\
\hline 7 & 7.40 & .01 & 296 & 235 & 61 \\
\hline 8 & 5.27 & .05 & 621 & 498 & 123 \\
\hline 9 & 10.40 & .01 & 812 & 698 & 114 \\
\hline \multicolumn{6}{|l|}{$\begin{array}{c}\text { Animated } \\
\text { videos }\end{array}$} \\
\hline 1 & 2.85 & .10 & 480 & 442 & 38 \\
\hline 2 & 2.77 & .10 & 539 & 498 & 41 \\
\hline 3 & 3.60 & .10 & 532 & 489 & 43 \\
\hline 4 & 6.70 & .01 & 424 & 401 & 23 \\
\hline
\end{tabular}

Note that 25 frames correspond to approximately one second in duration.

Table 2. Types of hazards in actual traffic clips

\begin{tabular}{|c|c|c|}
\hline Clips & Hazard Definition & Typical Characteristic \\
\hline 1 & $\begin{array}{l}\text { Motorcycle crossing the divided major road from the } \\
\text { opposite lane }\end{array}$ & $\begin{array}{l}\text { Unexpected/Road Violation } \\
\text { Heavy traffic }\end{array}$ \\
\hline 2 & Pedestrian jumping on the road & Violation in night vision \\
\hline 3 & Long vehicle suddlenly changes the lane & Unexpected/Road Violation \\
\hline 4 & $\begin{array}{l}\text { A vehicle suddenly emerging from the joining lane } \\
\text { on right in snowy weather }\end{array}$ & $\begin{array}{l}\text { Unexpected violation under the } \\
\text { presence of shadowing }\end{array}$ \\
\hline 5 & A vehicle moving backward in front & $\begin{array}{l}\text { Unexpected Violation under the } \\
\text { presence of shadowing stimuli }\end{array}$ \\
\hline 6 & Faulty overtaking (own lane) & Road Violation \\
\hline 7 & $\begin{array}{l}\text { A vehicle emerging from the lane on right with a } \\
\text { distance }\end{array}$ & Road Violation \\
\hline 8 & Cyclist suddenly emerging from right hand side & $\begin{array}{l}\text { Unexpected / Road Violation/ Heavy } \\
\text { traffic }\end{array}$ \\
\hline 9 & Faulty overtaking from right hand side & Road Violation \\
\hline
\end{tabular}




\section{DISCUSSION}

We compared video clips formed on the basis of actual traffic scenes with the animated clips in terms of discriminating HPS of experienced and novice drivers. Overall, findings suggested that hazards in real-traffic videos are relatively better than those in animated videos in separating groups. Consistent with previous studies (e.g., Sexton, 2001), novice drivers performed slightly worse than experienced drivers in detecting developing hazards on the road. Our findings showed that specific hazards and dangers involving sudden violations of road users, especially faulty overtaking, are critical risk factors for novice drivers in Turkey. In future studies, road hazards that are critical for safety and reflect the common pattern of violations in a specific culture should be utilized in test materials. Considering that violations involve behaviors that are inconsistent with the expected rule-based behavioral patterns in traffic, and that novices are less likely to anticipate them, specific aberrant driving behaviors that create risk in a specific driving environment should be included in hazard perception test materials. As suggested by McKenna, Horswill, and Alexander (2006), hazard perception performance of novice drivers can be improved by anticipation training. However, the domains of anticipation should be specified considering the most common driving violations and errors in a given culture. Therefore, hazard perception test materials should consist of specific situations that help drivers form active "ifthen" type schemas in anticipating hazards. This study suggested that anticipating the other road users" "unexpected" violations, which is very common in the Turkish traffic environment, makes novice drivers vulnerable to road crashes.

In sum, the results suggested that novice drivers should be trained on the basis of hazards typical to their driving environment and the instruments developed for assessing HPS should include characteristics representing the culture-specific pattern of driver behavior.

\section{ACKNOWLEDGMENTS}

This study was supported by "The Scientific and Technological Research Council of Turkey, Research \& Development Support Program (TUBITAK TIDEB)" and Meteksan Sistem A.Ş. We like to thank Ömer Çetin Sudan for his contribution in selecting the video clips and accessing the novice drivers.

\section{REFERENCES}

Bjornskau, T., \& Sagberg, F. (2005). What do novice drivers learn during the first months of driving? Improved handling skills or improved road-user interaction? Paper presented at the $3^{\text {rd }}$ International Conference on Traffic and Transport Psychology, Nottingham, UK.

Brown, I.D. (1982). Exposure and experience are a confounded nuisance in research on driver behavior. Accident Analysis and Prevention, 14, 345-352.

Deery, H.A. (1999). Hazard and risk perception among young novice drivers. Journal of Safety Research, 30, 225-236.

Falkmer, T., \& Gregersen, N.P. (2001). Fixation patterns of learner drivers with or without cerebral palsy (CP) when driving in real traffic environments. Transportation Research Part F 4, 171-185. 
Farrand, P, \& McKenna, F.P. (2001). Risk perception in novice drivers: the relationship between questionnaire measures and response latency. Transportation Research Part F 4, 201-212.

Jonah, B.A. (1986). Accident risk and risk-taking behaviour among young drivers. Accident Analysis and Prevention, 18, 255-271.

McKenna, F.P., \& Crick, J. (1997). Developments in hazard perception. TRL Report 297. Transport Research Laboratory, Crowthorne.

Mayhew, D.R., Simpson, H.M., \& Pak, A. (2003). Changes in collision rates among novice drivers during the first moths of driving. Accident Analysis and Prevention, 35, 683-691.

McKenna, F.P., Horswill, M.S., \& Alexander, J.L. (2006). Does anticipation training affect drivers' risk taking? Journal of Experimental Psychology: Applied, 12, 1-10.

McKnight, A.J. \& McKnight, A. S. (2003). Young novice drivers: careless or clueless? Accident Analysis and Prevention, 35, 921-925.

Sagberg, F., \& Bjornskau, T. (2006). Hazard perception and driving experience among novice drivers. Accident Analysis and Prevention, 38, 407-414.

Sexton, B. (2001). Development of hazard perception testing. Proceedings of the Novice Drivers Conference. 26 July 2001, UK. Available at www.dft.gov.uk/stellent/groups/ dft_rdsafety/documents/page/dft_rdsafety_611030.hcsp. Retrieved from July 10, 2005.

Wallis, T.S.A., \& Horswill, M.S. (In press). Using fuzzy signal detection theory to determine why experienced and trained drivers respond faster than novices in a hazard perception test. Accident Analysis and Prevention. 